\title{
INTERPOLAÇÃO DE EFEMÉRIDES GPS
}

\author{
GPS ephemerides interpolation \\ ANGELA CRISTINA CARARO ${ }^{1}$ \\ LUIZ DANILO DAMASCENO FERREIRA ${ }^{2}$ \\ ${ }^{1}$ Pontifícia Universidade Católica do Paraná - PUCPR \\ Centro de Ciências Exatas e de Tecnologia - CCET \\ Rua Imaculada Conceição, 1155 - Prado Velho \\ CEP: 80215-901 - Curitiba - PR - Brasil \\ Email: angela.cararo@pucpr.br \\ ${ }^{2}$ Universidade Federal do Paraná - UFPR \\ Departamento de Geomática Curitiba \\ Curso de Pós-Graduação em Ciências Geodésicas \\ Jardim das Américas - Centro Politécnico \\ CEP- 80531-990 -Curitiba - PR - Brasil \\ luizdanilo@ufpr.br
}

\section{RESUMO}

Neste trabalho é aplicado o procedimento de interpolação ao caso das efemérides GPS, que são normalmente disponibilizadas em formato de tabelas, em intervalos de 5 ou 15 minutos. Algumas aplicações GPS necessitam de dados de efemérides a intervalos menores do que isto. Diversos métodos de interpolação têm sido usados para gerar posições e velocidades dos satélites GPS nos pontos intermediários das tabelas. Os métodos de interpolação abordados serão o polinomial e trigonométrico, e serão aplicados sobre as efemérides rápidas IGS, com 24 horas de dados, em intervalos de 5 minutos, contendo a posição (coordenadas $\mathrm{x}, \mathrm{y} \mathrm{e} \mathrm{z}$ ) dos satélites GPS em dois sistemas de coordenadas: geocêntrico rotacional (ou terrestre) e geocêntrico inercial. Testes para determinar o número ótimo de termos nas interpolações foram conduzidos. Os dois tipos de funções utilizadas se mostraram adequadas, mas a interpolação através de funções trigonométricas mostrou-se o método mais adequado a ser aplicado nas efemérides GPS disponibilizadas pelo IGS no formato SP3. Nesse caso o melhor desempenho do interpolador se deu com 7 termos, com as efemérides-fonte no sistema inercial. O melhor resultado para interpolação polinomial aconteceu no caso da função apresentar entre 9 e 11 termos. Palavras-chave: GPS; Interpolação; Efemérides. 


\section{ABSTRACT}

In this paper, the interpolation procedure is applied in the case of GPS ephemerides, usually available in tables, where the data is provided in 5 or 15 minutes interval. Some GPS applications need ephemerides data in less spaced intervals. Many interpolations methods have been used to generate GPS satellites position and velocity in intermediate points of the tables. The interpolation methods used in this work are the polynomial and trigonometric ones and they will be applied on the IGS rapid ephemerides, with 24 hour total data spaced each 5 minutes, containing GPS satellite positions ( $\mathrm{x}, \mathrm{y}$ and $\mathrm{z}$ coordinates) in two coordinate frames: Earth-centered fixed (ECF) and inertial frame. Tests for determining the optimum number of interpolation terms were made. Both function types applied in interpolation tests worked well, but trigonometric interpolation was the most appropriate when applied on GPS ephemerides available in the SP3 files from IGS. In this case, 7 terms in the interpolating function was the most accurate result with source ephemerides in the inertial frame. The most accurate result found for polynomial interpolation occurred with 9 and 11 terms in the interpolating function.

Keywords: GPS; Interpolation; Ephemeredes.

\section{INTRODUÇÃO}

Interpolação é um tema vasto e importante. Se uma função numérica $f$ é conhecida apenas em um conjunto de pontos $x_{k}$, pode-se aproximar $f$ por uma função simples $P$ (geralmente um polinômio) que apresente os mesmos valores nestes pontos. Para todos os pontos de abcissa $x_{k}$ em um intervalo [a,b], substituise o valor desconhecido $f(x)$ pelo valor conhecido $P(x)$ : é o princípio da interpolação. Um polinômio interpola uma função $f(x)$ se $P\left(x_{k}\right)=f\left(x_{k}\right)$, para $k=0,1$, $2, \ldots, x$.

Segundo RALSTON e RABINOWITZ (2001), a interpolação repousa no centro da análise numérica clássica. Há duas razões principais para isto: a primeira é que em cálculos manuais há sempre a necessidade de encontrar-se o valor de uma função em uma tabela. No caso de se precisar de valores da função em pontos nãotabulados, é necessário interpolar. Entretanto, as tabelas altamente acuradas com pequenos incrementos do argumento que são comuns atualmente são, comparativamente, de origem recente. Por isto, a análise numérica clássica desenvolveu um grupo de métodos altamente sofisticados para interpolação. Atualmente, com computadores digitais, obtêm-se diretamente os valores das funções sem a necessidade de interpolar valores de uma tabela. Então, na maioria das vezes, a necessidade de interpolar surge de situações experimentais, onde se conhece uma tabela de pontos, obtidos através de observações ou experimentos, e deseja-se obter uma expressão analítica de uma dada curva $y(x)$ que melhor se adapte a este conjunto de pontos.

A computação das posições dos satélites GPS (Global Positioning System) é de importância fundamental em todos os softwares de posicionamento GPS 
(HOREMUZ e ANDERSSON, 2006). Os dados necessários para esta computação podem ser fornecidos na forma das efemérides transmitidas ou precisas. As efemérides transmitidas são enviadas ao usuário como um conjunto de parâmetros transmitidos na mensagem de navegação dos satélites GPS. Estes parâmetros são atualizados aproximadamente a cada duas horas, e a acurácia das coordenadas transmitidas é em torno de 1 metro. Há vários tipos de efemérides precisas (pósprocessadas) produzidas por várias agências globalmente distribuídas, baseadas nos dados das estações permanentes de monitoramento GPS. Sua acurácia pode variar desde $0,05 \mathrm{~m}$ para as preditas até $0,025 \mathrm{~m}$ para as finais, pós-processadas. As efemérides precisas são distribuídas no formato SP3 e estão disponíveis em http://igscb.jpl.nasa.gov/components/prods.html onde encontra-se uma tabela com todos os produtos IGS (International GNSS Service) e suas respectivas acurácia, latência, intervalos de dados e outras informações.

Neste trabalho será aplicado o procedimento de interpolação ao caso das efemérides $G P S$, que são normalmente disponibilizadas em formato de tabelas, em intervalos de 5 ou 15 minutos. Entretanto, algumas aplicações GPS necessitam de dados de efemérides a intervalos menores do que isto. Diversos métodos de interpolação têm sido usados para gerar posições e velocidades dos satélites GPS nos pontos intermediários das tabelas. Num primeiro momento, o método de Lagrange foi usado pelo US National Geodetic Survey (REMONDI, 1991). Interpolação polinomial e trigonométrica foram utilizadas por SCHENEWERK (2003). Os métodos de Lagrange, Chebyshev e trigonométrico foram realizados por FENG e ZHENG (2005). Interpolação sobre efemérides transmitidas, para aplicação em tempo real, foi implementada por HOREMUZ e ANDRESSON (2006). Para gerar tabelas interpoladas de efemérides com alta densidade de dados, YOUSIF e EL-RABBANY (2007) utilizaram os métodos de Lagrange, diferenças divididas de Newton, cubic spline e trigonométrico.

Os métodos de interpolação abordados neste trabalho serão o polinomial e trigonométrico, e serão aplicados sobre as efemérides rápidas, com 24 horas de dados, em intervalos de 5 minutos, contendo a posição (coordenadas $x, y$ e $z$ ) dos satélites GPS em dois sistemas de coordenadas: geocêntrico rotacional (ou terrestre) e geocêntrico inercial. O programa utilizado para gerar a interpolação é aquele desenvolvido por SCHENEWERK (2003) e disponibilizado em http://www.ngs.noaa.gov/gps-toolbox.

Testes para determinar o número ótimo de termos nas interpolações foram conduzidos. No capítulo 2 serão revistos brevemente alguns métodos mais comuns de interpolação. No capítulo 3 são apresentados o $I G S$ e seus serviços. No capítulo 4 é mostrada a metodologia utilizada no trabalho. Os resultados encontrados são apresentados na forma de gráficos e tabelas, no capítulo 5. As conclusões obtidas estão no capítulo 6 e, por fim, apresentam-se as referências. 


\section{ALGUNS MÉTODOS COMUNS DE INTERPOLAÇÃO}

Neste capítulo serão revisados brevemente alguns dos métodos mais utilizados para interpolação. Para maiores detalhes e apresentação de métodos mais sofisticados, consultar RALSTON e RABINOWITZ (2001), YOUNG e GREGORY (1972), KREYSZIG (2006).

\subsection{Polinômio Interpolador de $\mathbf{N}$ Pontos}

Considere-se uma família $F=A_{1}, \ldots, A_{n}$ de $n$ duplas $\left(x_{k}, y_{k}\right)$, os $x_{k}$ distintos. Dentro do caso freqüente onde os coeficientes $x_{k}$ e $y_{k}$ são reais, as duplas $A_{1}, \ldots, A_{N}$ formam uma nuvem de pontos num plano ( todos com abscissas distintas). Existe um polinômio único $P(F)$, de grau $\leq n-1$, tal que, para todo $k$ de $\{1, \ldots, n\}, P\left(x_{k}\right)=$ $y_{k} . P(F)$ é chamado de polinômio interpolador da família F. Há numerosas maneiras de se encontrar $P(F)$.

Esse conjunto de pontos $\left(x_{k}, y_{k}\right)$ pode advir de uma tabela de medidas físicas ou como pontos calculados de uma função $f(x)$ normalmente mais complicada de calcular, integrar ou diferenciar do que um polinômio. Neste caso pode-se comparar a função original $f(x)$ com a interpolada $p(x)$ e fazer uma estimativa dos erros de interpolação, da forma $|\mathrm{f}(\mathrm{x})-\mathrm{p}(\mathrm{x})|=\varepsilon$. Esse conjunto de pontos $\left(x_{k}, y_{k}\right)$ pode advir de uma tabela de medidas físicas ou como pontos calculados de uma função $f(x)$ normalmente mais complicada de calcular, integrar ou diferenciar do que um polinômio. Neste caso pode-se comparar a função original $f(x)$ com a interpolada $p(x)$ e fazer uma estimativa dos erros de interpolação, da forma $|\mathrm{f}(\mathrm{x})-\mathrm{p}(\mathrm{x})|=\varepsilon$.

\subsection{Fórmula de Lagrange}

O primeiro método conduz à chamada fórmula de Lagrange.

Para todo $k$ inteiro compreendido entre $l$ e $n$, define-se o polinômio $L_{k}$ da seguinte forma (COSTA, 1993):

$$
L_{k}(x)=\prod_{j=1, j \neq k}^{n} \frac{x-x_{j}}{x_{k}-x_{j}} .
$$

Por exemplo, se $n=4$,

$$
L_{2}(x)=\frac{\left(x-x_{1}\right)\left(x-x_{3}\right)\left(x-x_{4}\right)}{\left(x_{2}-x_{1}\right)\left(x_{2}-x_{3}\right)\left(x_{2}-x_{4}\right)}
$$

Cada polinômio $L_{k}$ possui grau $n-1$.

Pode-se verificar as seguintes igualdades:

$$
\forall k, j \in\{1, \ldots, n\}, L_{k}\left(x_{j}\right)=\delta_{k j}=\left\{\begin{array}{c}
1 s e j=k \\
0 s e j \neq k
\end{array}\right\}
$$


Constata-se agora que o polinômio $P(x)=\sum_{k=1}^{n} y_{k} L_{k}(x)$, conhecido como Fórmula de Lagrange para obtenção do polinômio de interpolação, satisfaz o problema, onde os valores $y_{k}=f\left(x_{k}\right)$ são conhecidos.

De fato, $P$ é de grau inferior ou igual a $n-1$ e ele verifica as igualdades:

$$
\forall j \in\{1, . ., n\}, P\left(x_{j}\right)=\sum_{k=1}^{n} y_{k} L_{k}\left(x_{j}\right)=\sum_{k=1}^{n} y_{k} \delta_{k j}=y_{j}
$$

A forma de Lagrange de um polinômio de interpolação tem apenas um interesse teórico. Na prática, ela é raramente utilizada. Ela demanda um número muito grande de operações. Por outro lado, todos os cálculos devem ser refeitos se decide-se juntar um outro ponto de interpolação. O polinômio de interpolação de $n$ pontos pode ser escrito de uma forma mais econômica em tempo de cálculo, e que demanda modificações pequenas a cada vez que um novo ponto é adicionado. É a forma de Newton, que utiliza o princípio das "diferenças divididas".

\subsection{Diferenças Divididas e Fórmula de Newton}

Sendo dados $n+1$ pontos, onde os $x_{i}$ são distintos mas não necessariamente ordenados, procura-se um polinômio $p(x)$ de grau $n$ que satisfaça

$$
p\left(x_{i}\right)=y_{i} \text { para } i=0,1, \ldots, n \text {. }
$$

aso $\mathrm{n}=1$ : Um polinômio de grau 1 é uma reta que passa por $\left(x_{0}, y_{0}\right), \quad\left(x_{1}\right.$, $\left.y_{1}\right)$. É dado por:

$$
p(x)=y_{0}+\left(x-x_{0}\right) \frac{y_{1}-y_{0}}{x_{1}-x_{0}}
$$

aso $\mathrm{n}=2$ : Para obter um polinômio de grau 2 (uma parábola) que passe pelos três pontos $\left(x_{0}, y_{0}\right),\left(x_{1}, y_{1}\right),\left(x_{2}, y_{2}\right)$, adiciona-se um termo de correção (de grau 2$)$ à equação (6). Como este termo deverá ser zero nos pontos $x_{0}$ e $x_{1}$,têm-se, necessariamente:

$$
p(x)=y_{0}+\left(x-x_{0}\right) \frac{y_{1}-y_{0}}{x_{1}-x_{0}}+a \cdot\left(x-x_{0}\right)\left(x-x_{1}\right) .
$$

$\mathrm{O}$ coeficiente $a$ é determinado por $p\left(x_{2}\right)=y_{2}$. Um cálculo simples (subtrair $p\left(x_{1}\right)$ de $p\left(x_{2}\right)$ e dividir por $\left(x_{2}-x_{0}\right)\left(x_{2}-x_{1}\right)$ fornece:

$$
a=\frac{1}{x_{2}-x_{0}}\left(\frac{y_{2}-y_{1}}{x_{2}-x_{1}}-\frac{y_{1}-y_{0}}{x_{1}-x_{0}}\right) \text {. }
$$


Um exemplo para o caso $n=5$ com os $x_{i}$ não eqüidistantes é mostrado na figura 1. Para os casos onde $n>2$ as fórmulas apresentam-se mais complexas e é recomendável introduzir-se uma notação conveniente para simplificar as expressões como na equação (8).

Figura 1 - Polinômio de interpolação de grau 5.

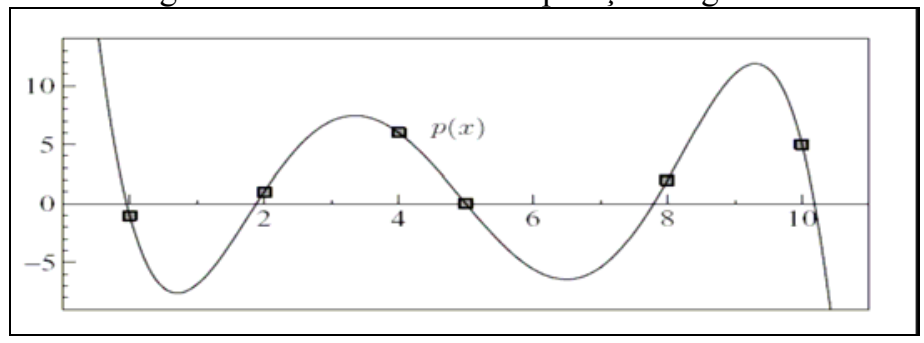

Definição (diferenças divididas): Para $\left(x_{i}, y_{i}\right)$ dados ( $x_{i}$ distintos) define-se

$$
\begin{aligned}
y\left[x_{i}\right] & :=y_{i} \\
\delta y\left[x_{i}, x_{j}\right] & :=\frac{y\left[x_{j}\right]-y\left[x_{i}\right]}{x_{j}-x_{i}} \\
\delta^{2} y\left[x_{i}, x_{j}, x_{k}\right] & :=\frac{\delta y\left[x_{j}, x_{k}\right]-\delta y\left[x_{i}, x_{j}\right]}{x_{k}-x_{i}} \\
\delta^{n} y\left[x_{i_{0}}, x_{i_{1}}, \ldots, x_{i_{n}}\right] & :=\frac{\delta^{n-1} y\left[x_{i_{1}, \ldots, i_{n}}\right]-\delta^{n-1} y\left[x_{i_{0}}, \ldots, x_{i_{n-1}}\right]}{x_{i_{n}}-x_{i_{0}}}
\end{aligned}
$$

Teorema (fórmula de Newton): O polinômio de interpolação de grau $n$ que passa pelos $n+1$ pontos $\left(x_{0}, y_{0}\right),\left(x_{1}, y_{1}\right), \ldots,\left(x_{n}, y_{n}\right)$, onde os $x_{i}$ são distintos, é único e é dado por

$$
\begin{aligned}
& p(x)=y\left[x_{0}\right]+\left(x-x_{0}\right) \delta y\left[x_{0}, x_{1}\right]+\left(x-x_{0}\right)\left(x-x_{1}\right) \delta^{2} y\left[x_{0}, x_{1}, x_{2}\right] \\
& +\ldots+\left(x-x_{0}\right)\left(x-x_{1}\right) \cdot \ldots \cdot\left(x-x_{n-1}\right) \delta^{n} y\left[x_{0}, x_{1}, \ldots, x_{n}\right] .
\end{aligned}
$$

Demonstração: Para $n=1$ e $n=2$ a equação (13) é equivalente a equação (6) e as equações (7) e (8). Para demonstrar o caso geral, procede-se por recorrência. Supõe-se que:

$$
p_{1}(x)=y\left[x_{0}\right]+\left(x-x_{0}\right) \delta y\left[x_{0}, x_{1}\right]+\ldots+\left(x-x_{0}\right) \cdot \ldots \cdot\left(x-x_{n-2}\right) \delta^{n-1} y\left[x_{0}, x_{1}, \ldots, x_{n-1}\right]
$$

seja o polinômio único de grau $n-1$ que passa por $\left(x_{i}, y_{i}\right)$ para $i=0,1, \ldots, n-1$. Então, como na equação (7), o polinômio $p(x)$ é necessariamente da forma 


$$
p(x)=p_{1}(x)+a \cdot\left(x-x_{0}\right)\left(x-x_{1}\right) \cdot \ldots \cdot\left(x-x_{n-1}\right),
$$

onde $a$ é determinado por $p\left(x_{n}\right)=y_{n}$. Isto resulta na unicidade do polinômio de interpolação.

Para mostrar que $a=\delta^{n} y\left[x_{0}, x_{1}, \ldots, x_{n}\right]$, o que finaliza a demonstração, considera-se igualmente o polinômio de grau $n-1$ :

$$
p_{2}(x)=y\left[x_{1}\right]+\left(x-x_{1}\right) \delta y\left[x_{1}, x_{2}\right]+\ldots+\left(x-x_{1}\right) \cdot \ldots \cdot\left(x-x_{n-1}\right) \delta^{n-1} y\left[x_{1}, x_{2}, \ldots, x_{n}\right]
$$

que passa por $\left(x_{i}, y_{i}\right)$ para $i=1, \ldots, n$. Depois, afirma-se (idéia de Aitken-Neville, 1929, 1932)

$$
q(x):=\frac{1}{x_{n}-x_{0}}\left(\left(x_{n}-x\right) p_{1}(x)+\left(x-x_{0}\right) p_{2}(x)\right) .
$$

Trata-se de um polinômio de grau $n$ que satisfaz a equação (5) para o ponto $x_{0}$ (aqui, o fator $\left(x-x_{0}\right)$ é nulo), e para os pontos $x_{i}, i=1, \ldots, n-1$ (aqui, os dois polinômios $p_{l}(x)$ e $p_{2}(x)$ são iguais a $y_{i}$. Pela unicidade do polinômio de interpolação temos agora $q(x)=p(x)$. Comparando o coeficiente de $x^{n}$ da equação (17) com aquele da equação (15) obtêm-se:

$$
a=\frac{1}{x_{n}-x_{0}}\left(\delta^{n-1} y\left[x_{1}, \ldots, x_{n}\right]-\delta^{n-1} y\left[x_{0}, \ldots, x_{n-1}\right]\right)=\delta^{n} y\left[x_{0}, \ldots, x_{n}\right]
$$

o que demonstra a equação (13).

Tabela 1 - Diferenças divididas para os dados da figura 1.

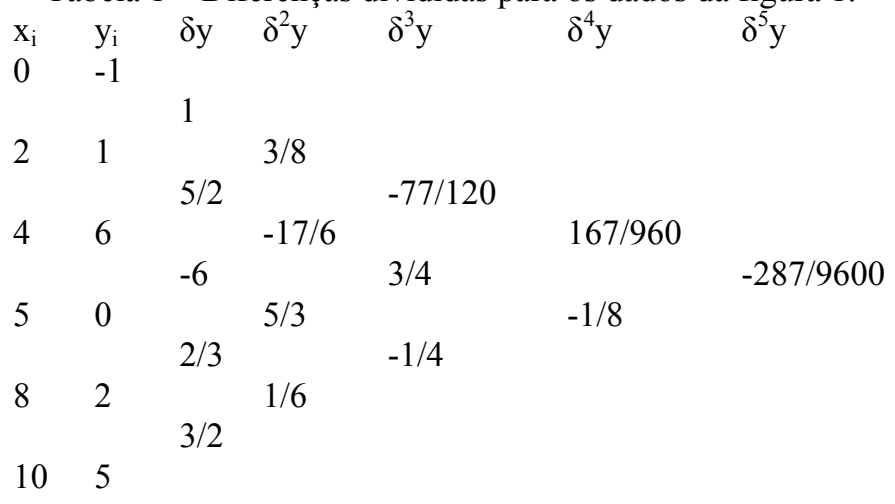

Exemplo: Para os dados da figura 1, as diferenças divididas são apresentadas na tabela 1 . O polinômio de interpolação é dado então por: 


$$
\begin{aligned}
& p(x)=-1+x+x(x-2) \frac{3}{8}-x(x-2)(x-4) \frac{77}{120}+x(x-2)(x-4)(x-5) \frac{167}{960} \\
& -x(x-2)(x-4)(x-5)(x-8) \frac{287}{9600} .
\end{aligned}
$$

\subsection{O Algoritmo de Neville}

Há um outro método de encontrar o polinômio de interpolação $P$ de duas listas $X$ (abcissas) e $Y$ (ordenadas), útil sobretudo para calcular os valores deste polinômio: trata-se do Algoritmo de Neville (que se assemelha ligeiramente com o método das diferenças divididas).

Considera-se, por exemplo, os 3 pontos $A_{1}\left(x_{1}, y_{l}\right), A_{2}\left(x_{2}, y_{2}\right), A_{3}\left(x_{3}, y_{3}\right)$, de abcissas distintas. Seja $P_{l, 1}$ o polinômio de grau 0 "interpolando" somente $A_{l}$ : é a constante $y_{1}$.

Da mesma forma, sejam $P_{2,2}$ e $P_{3,3}$ os polinômios constantes "interpolando" respectivamente $A_{2}$ e $A_{3}$. Generalizando esta notação, seja $P_{1,2}$ o polinômio ( de grau inferior ou igual a 1 ) interpolando os dois pontos $A_{1}$ e $A_{2}$, e seja $P_{2,3}$ aquele que interpola $A_{2}$ e $A_{3}$. Constata-se em COSTA (1993) que:

$$
P_{1,2}=\frac{\left(X-x_{2}\right) x_{1}+\left(x_{1}-X\right) x_{2}}{x_{1}-x_{2}}=\frac{\left(X-x_{2}\right) P_{1,1}+\left(x_{1}-X\right) P_{2,2}}{x_{1}-x_{2}}
$$

Da mesma maneira:

$$
P_{2,3}=\frac{\left(X-x_{3}\right) x_{2}+\left(x_{2}-X\right) x_{3}}{x_{2}-x_{3}}=\frac{\left(X-x_{3}\right) P_{2,2}+\left(x_{2}-X\right) P_{3,3}}{x_{2}-x_{3}}
$$

Seja $P_{1,2,3}$ o polinômio, de grau inferior ou igual a 2 , interpolando os três pontos $A_{1}, A_{2}$ e $A_{3}$.

Constata-se enfim que:

$$
P_{1,2,3}=\frac{\left(X-x_{3}\right) P_{1,2}+\left(x_{1}-X\right) P_{2,3}}{x_{1}-x_{3}}
$$

Com efeito:

$$
P_{1,2,3}\left(x_{1}\right)=\frac{\left(x_{1}-x_{3}\right) P_{1,2}\left(x_{1}\right)}{x_{1}-x_{3}}=y_{1}, \quad P_{1,2,3}\left(x_{3}\right)=\frac{\left(x_{1}-x_{3}\right) P_{2,3}\left(x_{3}\right)}{x_{1}-x_{3}}=y_{3},
$$

$\mathrm{e}$

$$
P_{1,2,3}\left(x_{2}\right)=\frac{\left(x_{2}-x_{3}\right) P_{1,2}\left(x_{2}\right)+\left(x_{1}-x_{2}\right) P_{2,3}\left(x_{2}\right)}{x_{1}-x_{3}}=\frac{\left(x_{2}-x_{3}\right) y_{2}+\left(x_{1}-x_{2}\right) y_{2}}{x_{2}-x_{3}}=y_{2}
$$

Mais geralmente, seja uma família de $n$ pontos $A_{k}\left(x_{k}, y_{k}\right)$, de abcissas distintas duas à duas. Seja $P_{1,2, \ldots, n-1}$ o polinômio interpolando os $n-1$ primeiros pontos $A_{1}$, $A_{2}, \ldots, A_{n-1}$. Seja $P_{2,3, \ldots, n}$ o polinômio interpolando os $n-1$ últimos pontos $A_{2}, A_{3}, \ldots$, $A_{n}$. 
Estes dois polinômios são de grau inferior ou igual a $n-2$. O polinômio:

$$
P=\frac{\left(X-x_{n}\right) P_{1,2, \ldots, n-1}+\left(x_{1}-X\right) P_{2,3, \ldots, n}}{x_{1}-x_{n}}
$$

possui grau $\leq n-1$ e constata-se que ele interpola os $n$ pontos $A_{1}, A_{2}, \ldots, A_{n}$.

A partir dos n pontos, aplica-se então o método seguinte:

- Escrever os $n$ polinômios constantes $P_{1,1}, P_{2,2}, \ldots, P_{n, n}$ "interpolando" cada um dos pontos.

- Associar duas a duas estas constantes para formar os $n-1$ polinômios $P_{1,2}$, $P_{2,3}, \ldots, P_{n-1, n}$ interpolando dois pontos consecutivos da família.

- Associar estes polinômios dois a dois para formar os $n-2$ polinômios $P_{1,2,3}$, $P_{2,3,4}$, etc., interpolando três pontos consecutivos da família.

- Prosseguir este procedimento até o polinômio $P=P_{1,2, \ldots, n}$ interpolando os $n$ pontos da família.

- A cada etapa, todo novo polinômio interpolador é formado a partir de dois "parentes", e o fato que as formulas de cálculo são as mesmas (nos índices próximos) permite escrever um algoritmo simples.

Segue um exemplo do algoritmo de Neville para o caso de uma família de 5 pontos. Por economia de espaço não serão mostradas as duas últimas colunas, que terminam a tabela 2 (triangular) para o cálculo do polinômio de interpolação $\mathrm{P}_{1,2,3,4,5}$ destes 5 pontos.

Tabela 2 - Exemplo do algoritmo de Neville para família de 5 pontos

$$
\begin{array}{lll}
P_{1,1}=y_{1} & \\
& P_{1,2}=\frac{\left(\mathrm{X}-x_{2}\right) P_{1,1}+\left(x_{1}-\mathrm{X}\right) P_{2,2}}{x_{1}-x_{2}} & \\
P_{2,2}=y_{2} & P_{1,2,3}=\frac{\left(\mathrm{X}-x_{3}\right) P_{1,2}+\left(x_{1}-\mathrm{X}\right) P_{2,3}}{x_{1}-x_{3}} \\
P_{3,3}=y_{3} & P_{2,3}=\frac{\left(\mathrm{X}-x_{3}\right) P_{2,2}+\left(x_{2}-\mathrm{X}\right) P_{3,3}}{x_{2}-x_{3}} & P_{2,3,4}=\frac{\left(\mathrm{X}-x_{4}\right) P_{2,3}+\left(x_{2}-\mathrm{X}\right) P_{3,4}}{x_{2}-x_{4}} \\
P_{4,4}=y_{4} & P_{3,4}=\frac{\left(\mathrm{X}-x_{4}\right) P_{3,3}+\left(x_{3}-\mathrm{X}\right) P_{4,4}}{x_{3}-x_{4}} & P_{3,4,5}=\frac{\left(\mathrm{X}-x_{5}\right) P_{3,4}+\left(x_{3}-\mathrm{X}\right) P_{4,5}}{x_{3}-x_{5}} \\
P_{5,5}=y_{5} & P_{4,5}=\frac{\left(\mathrm{X}-x_{5}\right) P_{4,4}+\left(x_{4}-\mathrm{X}\right) P_{5,5}}{x_{4}-x_{5}} &
\end{array}
$$

\subsection{Interpolação Trigonométrica}

Considere-se uma aplicação numérica $f$ ( definida sobre o conjunto dos números reais) e periódica de período $2 \pi$. Supõe-se que são conhecidos apenas os valores de $f$ em $\boldsymbol{n}$ pontos do segmento $[0,2 \pi]$ (ou de outro intervalo de comprimento $2 \pi)$.

Deseja-se estimar os valores de $f$ em um ponto qualquer deste intervalo. Para isto, procura-se uma função trigonométrica $g$ (que também possui período igual a 
$2 \pi$ ) o mais "simples" possível, apresentando o mesmo valor que $f$ em cada um destes $n$ pontos.

A função $g$ será então encarregada de interpolar $f$ sobre o restante do intervalo.

O modelo é aquele das combinações lineares das funções $x \rightarrow \exp (i k x)$, para $k$ inteiro. Para minimizar as freqüências utilizadas, é razoável centrar os valores dos inteiros $k$ em torno da origem. Ter-se-ão $n$ coeficientes desconhecidos pois será resolvido um problema de interpolação a $n$ pontos.

Isto conduz a função $g$ seguinte (o intervalo de variação do índice $k$ leva em conta a paridade de $n$ ):

$$
g:(n, x) \mapsto \sum_{k=\left[\frac{-n}{2}\right]+1}^{\left[\frac{n}{2}\right]} a_{k} \exp (i k x)
$$

Por exemplo, se $n$ é impar, $(n=2 q+1)$ :

$$
g(n, x)=a_{-q} \exp (-i q x)+\ldots+a_{-1} \exp (-i x)+a_{0}+a_{1} \exp (i x)+\ldots+a_{q} \exp (i q x)
$$

E se $n$ é par $(n=2 q)$ :

$$
g(n, x)=a_{-q+1} \exp (-i(-q+1) x)+\ldots+a_{-1} \exp (-i x)+a_{0}+a_{1} \exp (i x)+\ldots+a_{q} \exp (i q x)
$$

Em todos os casos, $g(n, x)$ é uma soma de $n$ termos.

Associa-se a $g$ o polinômio seguinte:

$$
P:(n, X) \mapsto \sum_{k=0}^{n-1} a_{k+1+[-n / 2]} X^{k}
$$

Assim, quando $n$ é impar $(n=2 q+1)$ :

$$
P(n, X)=a_{-q}+a_{-q+1} X+\ldots+a_{q-1} X^{n-2}+a_{q} X^{n-1} .
$$

Se $n$ é $\operatorname{par}(n=2 q)$ :

$$
P(n, X)=a_{-q+1}+a_{-q+2} X+\ldots+a_{q-1} X^{n-2}+a_{q} X^{n-1} .
$$

Constata-se que para $x$, com $m=[-n / 2]+1$, e colocando $\omega=\exp (i x)$ : 


$$
g(n, x)=\sum_{k=m}^{\left[\frac{n}{2}\right]} a_{k} \omega^{k}=\omega^{m} \sum_{k=m}^{\left[\frac{n}{2}\right]} a_{k} \omega^{k-m}=\omega^{m} \sum_{k=0}^{n-1} a_{k+m} \omega^{k}=\omega^{m} P(n, \omega)
$$

O problema da interpolação trigonométrica dos $n$ pontos $A_{j}=\left(x_{j}, y_{j}\right)$ pode ser colocado na seguinte forma: trata-se de encontrar os $n$ coeficientes $a_{k}$ tal que $\forall j=0, \ldots, n-1: g\left(n, x_{j}\right)=y_{j}$.

Quando se associa a aplicação polinomial $P$ à função trigonométrica $g$, retorna-se a um problema de interpolação polinomial. Trata-se, efetivamente, de encontrar os coeficientes $a_{k}$ do polinômio $P(\operatorname{deg}(P) \leq n-1)$ tal que:

$$
\forall j=0, \ldots, n-1: P\left(n, \omega_{j}\right)=\omega_{j}^{-m} y_{j}, \operatorname{com} m=1+\left[-\frac{n}{2}\right] \quad \text { e } \omega_{j}=\exp \left(i x_{j}\right) \cdot
$$

Quando o problema é resolvido (ou seja, quando os coeficientes do polinômio são encontrados) então a função $g$ pode ser avaliada em um ponto qualquer $x$, escrevendo-se:

$$
\forall x \in R, \quad g(n, x)=\omega_{x}^{m} P\left(n, \omega_{x}\right), \quad \operatorname{com} \quad \omega_{x}=\exp (i x) \quad \text { e } \quad m=1+\left[-\frac{n}{2}\right] .
$$

\section{EFEMÉRIDES PRECISAS DOS SATÉLITES GPS E INFORMAÇÃO SOBRE OS RELÓGIOS}

International GNSS Service - IGS (antigamente: International GPS Service) foi formalmente estabelecido pela International Association of Geodesy (IAG) em 1993 e iniciou oficialmente suas operações em $1^{\circ}$. de janeiro de 1994 . O IGS gera e dissemina efemérides precisas para os satélites GPS e também fornece produtos relacionados como Earth Orientation Parameters $(E O P)$ e correções dos relógios para os satélites. Os serviços $I G S$ estão baseados em uma rede global de monitoramento e estações de rastreamento GNSS e fornece informações e dados de seus centros de processamento de dados para todos os usuários GNSS.

Os arquivos com informações orbitais dos satélites $G P S$, que são fornecidos pelo IGS, estão no formato definido pelo National Geodetic Survey (NGS), o chamado SP3. Este é uma representação ASCII que inclui as posições dos satélites e correções dos relógios.

Este formato foi cuidadosamente planejado para levar em consideração também seu uso para o GLONASS e para satélites geoestacionários. Enquanto a primeira geração deste formato podia acomodar somente 35 satélites, a segunda geração pode acomodar até 85 satélites. $O$ cabeçalho da segunda geração foi melhorado para receber modificações e novas informações como, por exemplo, a acurácia da informação orbital de cada satélite (REMONDI, 1991).

O formato SP3 é dado em unidades de Km para as coordenadas e em microssegundos para o erro dos relógios dos satélites, com acurácia de $1 \mathrm{~mm}$ e 1 ps (pico-segundo), respectivamente. Se os arquivos com as velocidades dos satélites estão incluídos, (formato SP1), as unidades para velocidade são 
decímetros/segundo, com uma acurácia de $10^{-4} \mathrm{~mm} / \mathrm{s}$ ). A variação na marcha dos relógios (rate of change) é dada em unidades de $10^{-4} \mathrm{ps} / \mathrm{s}$ (HILLA, 2007).

As informações sobre os relógios dos satélites, no formato SP3 são fornecidas nas mesmas épocas (a cada 5 ou $15 \mathrm{~min}$ ) para as quais são também fornecidas as posições dos satélites. A interpolação é necessária para obter-se a posição do satélite e a correção do relógio na época de transmissão.

\section{METODOLOGIA UTILIZADA}

SCHENEWERK (2003) usa as duas estratégias mais comuns de interpolação de efemérides GPS: interpolação polinomial e interpolação por funções trigonométricas.

Na interpolação polinomial :

$$
C=A_{0}+A_{1} T+A_{2} T^{2}+A_{3} T^{3}+\ldots+A_{N} T^{N}
$$

No primeiro caso, um simples polinômio é a função analítica que irá aproximar o conjunto de dados no interpolador. Aqui, $C$ representa uma das coordenadas $(X, Y$ ou $Z)$ da posição do satélite $G P S$, em um sistema de referências geocêntrico inercial ou rotacional (terrestre), $T$ é o tempo, e $A_{0}$ até $A_{N}$ são os coeficientes que deverão ser encontrados pelo processo de interpolação para representar o conjunto de dados de entrada (efemérides-fonte). O algoritmo usado é o de Neville.

No segundo caso, a escolha baseia-se no comportamento naturalmente cíclico das coordenadas da posição do satélite GPS. A figura 2 mostra o gráfico das coordenadas $x, y$ e $z$ da posição do satélite GPS PRN 01, num sistema de referência geocêntrico rotacional ( $I G S$ 2000), para o dia 01.01.2002, obtido à partir das efemérides rápidas igr11472.sp3, fornecidas pelo IGS.

Figura 2 - Gráfico das coordenadas do PRN 01, para o dia 01.01.2002, no sistema de referência geocêntrico rotacional. Fonte: (SCHENEWERK, 2003).

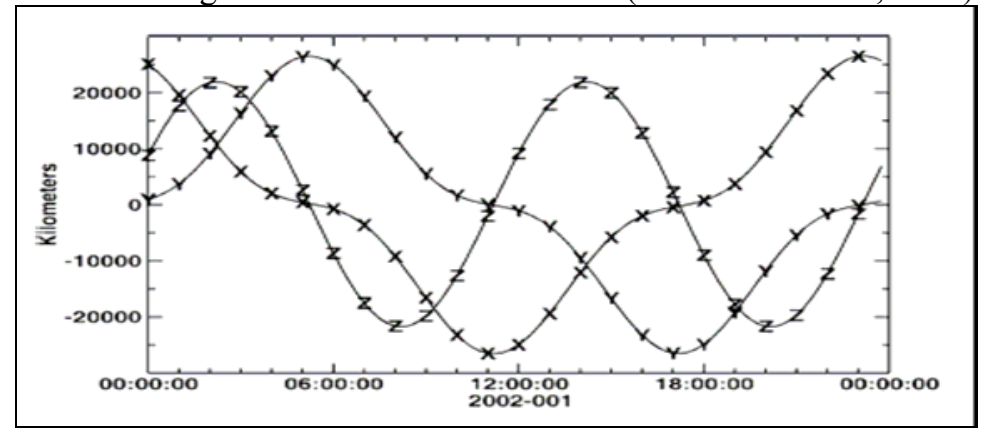

Deve-se observar que as três coordenadas apresentam comportamento cíclico, mas apenas a coordenada $z$ possui o período esperado de 12 horas. As coordenadas $x$ e $y$ apresentam um comportamento mais complexo, de período aproximado de 24 
horas. Isto ocorre em virtude destas coordenadas estarem expressas num sistema referencial geocêntrico rotacional (IGS2000), combinando, assim, o movimento orbital dos satélites com o da rotação da Terra. Para o caso das coordenadas dos satélites estarem representadas num sistema geocêntrico inercial, o comportamento das coordenadas $\mathrm{x}, \mathrm{y}$, e $\mathrm{z}$ é aproximadamente senoidal com período de 12 horas, conforme mostra a figura 3 .

Figura 3 - Gráfico das coordenadas do PRN 01, para o dia 01.01.2002, expressas no sistema de referência geocêntrico inercial. Fonte: SCHENEWERK, 2003.

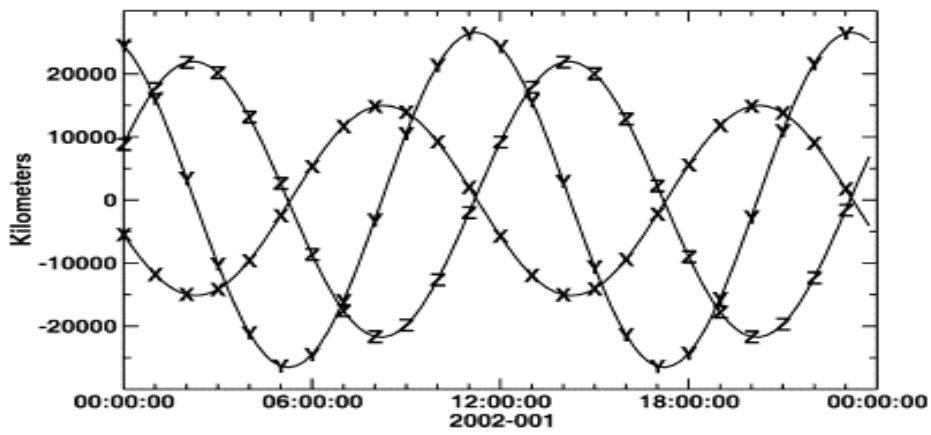

SCHENEWERK (2003) disponibiliza, através da página do NOAA (http://www.ngs.noaa.gov/gps-toolbox) o programa executável em linguagem C (atest) para realizar os dois casos de interpolação: polinomial (através do algoritmo de Neville) e trigonométrica (distinguindo-se o caso das efemérides-fonte estarem expressas em sistema inercial ou rotacional). Disponibiliza também um conjunto de dados da seguinte forma:

- Efemérides fonte: estruturada da mesma forma que as efemérides (final ou rápida) típicas do $I G S$, no formato $\mathrm{SP} 3$, consistindo em uma tabela com dados das coordenadas $x, y$ e $z$, da posição dos 32 satélites (e das correções dos relógios) a cada 15 minutos iniciando às 00:00:00 até 23:45:00 GPS Time, para o dia em questão.

- Efemérides de controle: também estão no formato SP3, mas as coordenadas dos satélites estão apresentadas a cada 5 minutos.

- Estas são as tabelas (efemérides-fonte e de controle) que serão utilizadas para os testes com os interpoladores.

Com o programa atest é possível "entrar" com:

- as efemérides fonte e controle,

- o número $P R N$ do satélite GPS,

- o número de pontos (dados) centrados em cada época, usado para computar os coeficientes de interpolação,

- o número de termos a ser usado na função de interpolação, 
- e um caracter especificando a função (algoritmo).

\section{RESULTADOS E ANÁLISES}

Nos testes realizados foi analisado o desempenho dos interpoladores. Foram recriados e estendidos os resultados obtidos por SCHENEWERK (2003). Os dados para teste, incluindo as efemérides fonte e de referência foram obtidos do autor e foram seguidos critérios similares de análise. Nestas análises, o número de pontos usados foi igual ao número de coeficientes a serem determinados, em todos os casos. Os valores mostrados nas tabelas 3 e 5 representam os resultados encontrados para os procedimentos de interpolação (polinomial e trigonométrico), aplicados aos dados de entrada (efemérides-fonte) do satélite PRN 01, para o dia 01.01.2002. A coluna indicada com "Max" é a máxima diferença encontrada (no módulo do vetor posição) entre os valores interpolados e aqueles fornecidos pelas efemérides de controle. Na tabela 3 as efemérides-fonte estavam expressas no sistema geocêntrico rotacional e na tabela 5 no sistema geocêntrico inercial. As tabelas 4 e 6 resumem os mesmos tipos de resultados, no caso do satélite GPS PRN 18.

Tabela 3 - Máximo desvio da função interpolada representando as efemérides geocêntricas, rotacionais, para o PRN 01, em função do número de termos usados na função.

\begin{tabular}{|c|c|c|}
\hline \multirow[t]{2}{*}{ Termos } & Polinomial & Trigonométrico \\
\hline & Max. (cm) & Max.(cm) \\
\hline 5 & 184738,1 & 82512,9 \\
\hline 7 & 6615,0 & 303,5 \\
\hline 9 & 248,1 & 10,3 \\
\hline 11 & 72,4 & 61,7 \\
\hline 13 & 269,3 & 242,4 \\
\hline 15 & 1017,3 & 863,6 \\
\hline
\end{tabular}

Tabela 4 - Máximo desvio da função interpolada representando as efemérides geocêntricas, rotacionais, para o PRN 18, em função do número de termos usados na função.

\begin{tabular}{|c|c|c|}
\hline \multirow[t]{2}{*}{ Termos } & Polinomial & Trigonométrico \\
\hline & Max. (cm) & Max.(cm) \\
\hline 5 & 184261,4 & 84098,9 \\
\hline 7 & 6859,7 & 164,0 \\
\hline 9 & 282,2 & 13,1 \\
\hline 11 & 53,5 & 48,6 \\
\hline 13 & 260,0 & 234,3 \\
\hline 15 & 964,5 & 818,9 \\
\hline
\end{tabular}

Bol. Ciênc. Geod., sec. Artigos, Curitiba, v. 17, nº 2, p.218-238, abr-jun, 2011. 
Tabela 5 - Máximo desvio da função interpolada representando as efemérides geocêntricas, inerciais, para o PRN 01, em função do número de termos usados na função.

\begin{tabular}{lrr}
\hline Termos & \multicolumn{1}{c}{ Polinomial } & Trigonométrico \\
\cline { 2 - 3 } & Max. (cm) & Max.(cm) \\
\cline { 3 - 3 } & & 70,1 \\
\cline { 3 - 3 } 7 & 101162,1 & 8,2 \\
9 & 1780,6 & 14,5 \\
11 & 41,7 & 29,4 \\
13 & 38,8 & 103,8 \\
15 & 155,4 & 428,2 \\
\hline
\end{tabular}

Tabela 6 - Máximo desvio da função interpolada representando as efemérides geocêntricas, inerciais, para o PRN 18, em função do número de termos usados na função.

\begin{tabular}{|c|c|c|}
\hline \multirow[t]{2}{*}{ Termos } & Polinomial & Trigonométrico \\
\hline & Max. (cm) & Max.(cm) \\
\hline 5 & 106405,2 & 105,5 \\
\hline 7 & 2040,9 & 10,1 \\
\hline 9 & 39,2 & 14,7 \\
\hline 11 & 57,9 & 44,0 \\
\hline 13 & 282,6 & 180,6 \\
\hline 15 & 1116,9 & 556,9 \\
\hline
\end{tabular}

A figura 4 mostra as diferenças, em $\mathrm{cm}$, entre os valores de referência e os interpolados, ao longo do dia 01.01.2002, para o PRN 01. Aqui o método de interpolação é o trigonométrico ( 7 termos) com efemérides-fonte no sistema geocêntrico inercial.

'A figura 5 mostra as diferenças, em $\mathrm{cm}$, entre os valores de referência e os interpolados, ao longo do dia 01.01.2002, para o PRN 01. Aqui o método de interpolação é o polinomial ( 5 termos) com efemérides-fonte no sistema geocêntrico rotacional. 
Figura 4 - Diferenças entre os valores de referência e interpolados, para o PRN 01, dia 01.01.2002, no caso de interpolação trigonométrica ( 7 termos) sobre efemérides-fonte no sistema geocêntrico inercial.

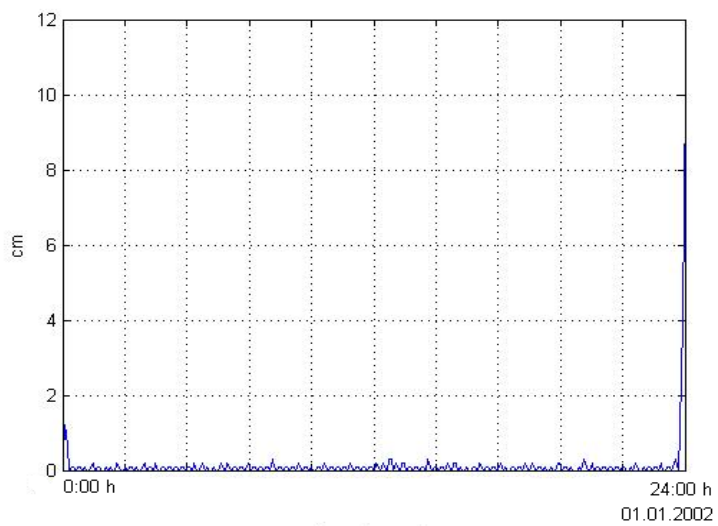

Figura 5 - Diferenças entre os valores de referência e interpolados, para o PRN 01, dia 01.01.2002, no caso de interpolação polinomial ( 5 termos) sobre efeméridesfonte no sistema geocêntrico rotacional.

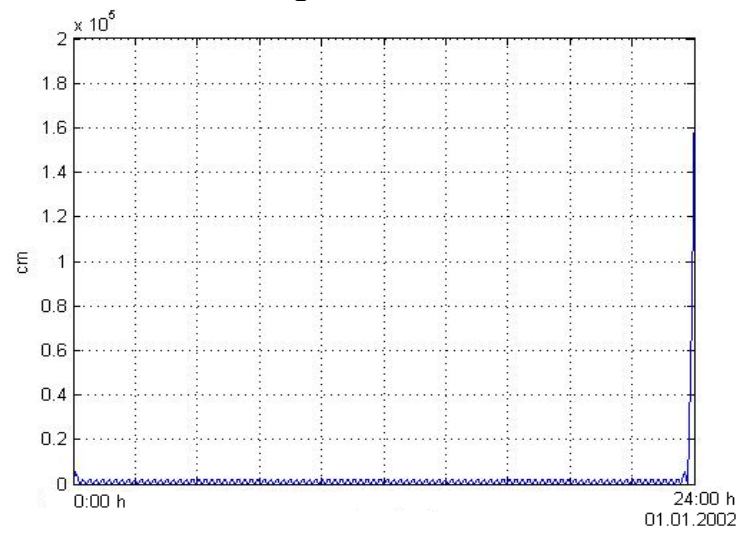

$\mathrm{Na}$ figura 6 são mostradas as diferenças entre coordenadas interpoladas e de controle, em função do tempo, para o PRN 01. O horário de início é 23:00 horas e término 24:00 horas, para o dia 01.01.2002. Neste caso, a interpolação realizada é a trigonométrica, com efemérides-fonte no sistema rotacional, com 9 termos na função interpolada.

$\mathrm{Na}$ figura 7 são mostradas as diferenças entre coordenadas interpoladas e de controle, em função do tempo, para o PRN 01. O horário de início é 23:00 horas e 
término 24:00 horas, para o dia 01.01.2002. Neste caso, a interpolação realizada é a polinomial, com efemérides-fonte no sistema rotacional, com 11 termos na função interpolada.

Figura 6 - Desempenho do interpolador, para o PRN 01, na interpolação trigonométrica sobre efemérides-fonte no sistema rotacional, no caso da função interpolada com 9 termos.

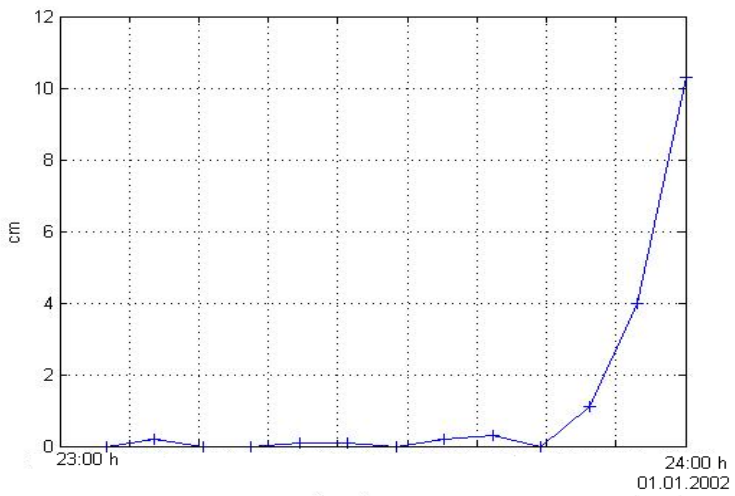

Figura 7 - Desempenho do interpolador, para o PRN 01, na interpolação polinomial sobre efemérides-fonte no sistema rotacional, no caso da função interpolada com 11 termos.

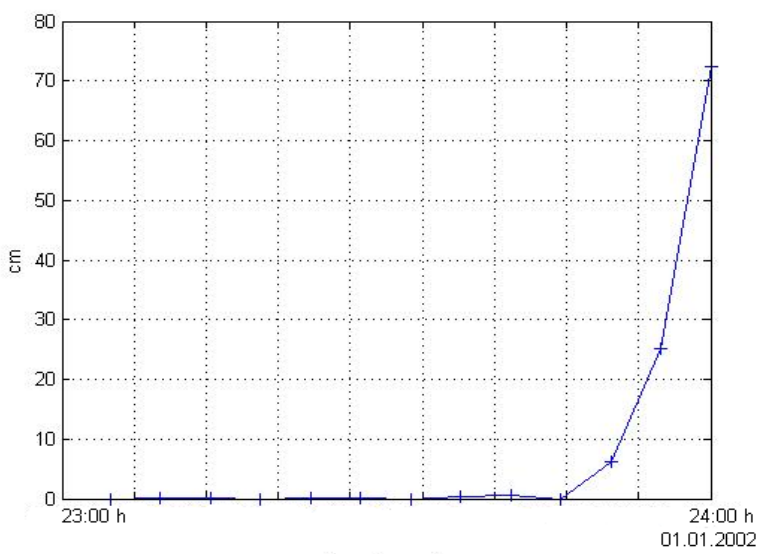

$\mathrm{Na}$ figura 8 são mostradas as diferenças entre coordenadas interpoladas e de controle, em função do tempo, para o PRN 01. O horário de início é 23:00 horas e término 24:00 horas, para o dia 01.01.2002. Neste caso, a interpolação realizada é a trigonométrica, com efemérides-fonte no sistema inercial, com 7 termos na função interpolada.

Bol. Ciênc. Geod., sec. Artigos, Curitiba, v. 17, nº 2, p.218-238, abr-jun, 2011. 
Figura 8 - Desempenho do interpolador, para o PRN 01, na interpolação trigonométrica sobre efemérides-fonte no sistema inercial, no caso da função interpolada com 7 termos.

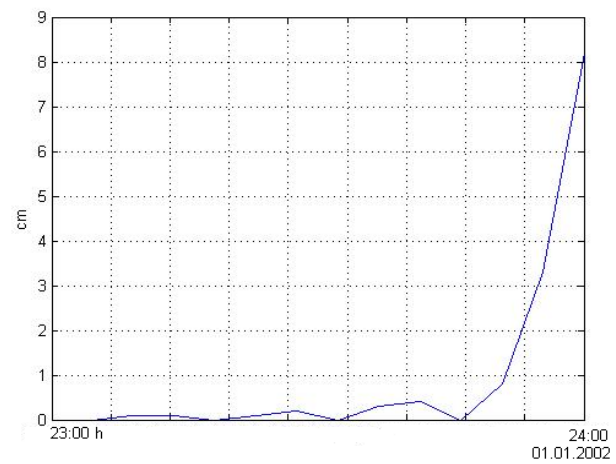

Na figura 9, são mostradas as diferenças entre coordenadas interpoladas e de controle, em função do tempo, para o PRN 01. O horário de início é 23:00 horas e término 24:00 horas, para o dia 01.01.2002. Neste caso, a interpolação realizada é a polinomial, com efemérides-fonte no sistema inercial, com 9 e 11 termos nas funções interpoladas.

Figura 9 - Desempenho do interpolador, para o PRN 01, na interpolação polinomial sobre efemérides-fonte no sistema inercial, no caso da função interpolada com $9 \mathrm{e}$ 11 termos.

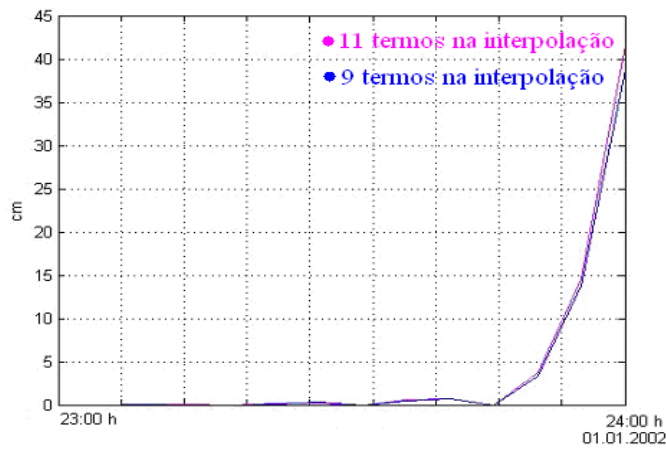

Através da análise das tabelas e gráficos apresentados neste trabalho, pode-se inferir o que segue:

- Para os primeiros 15 minutos e últimos 15 minutos dos dados (ver figuras 4 e 5) observa-se um efeito de degeneração do procedimento de interpolação. Isto está ligado ao fenômeno de Runge (maior oscilação da 
função interpoladora nas bordas da interpolação), típico nestes procedimentos e também à natureza dos dados. Como os erros de interpolação são mais evidentes ao final do dia, as figuras seguintes enfatizaram este aspecto.

- A precisão nominal das efemérides $G P S$, fornecidas pelo $I G S$, para a época dos dados, é de $3 \mathrm{~cm}$ para as rápidas e $2 \mathrm{~cm}$ para as finais, pósprocessadas. Dois tipos de funções foram testadas para representar as efemérides-fonte (rápidas) e ambas se mostraram bastante adequadas para interpolação assumindo-se um número apropriado de termos na função a ser usada.

- Das tabelas e gráficos apresentados verifica-se que, no caso de efeméridesfonte no sistema rotacional, o melhor resultado para interpolação polinomial aconteceu no caso da função apresentar entre 9 e 11 termos. E o melhor resultado para a interpolação trigonométrica foi no caso da função apresentar 9 termos.

- Verifica-se, também, através das tabelas e gráficos, que, no caso de efemérides-fonte no sistema inercial, o melhor resultado apresentado pelo interpolador polinomial foi também para 9 e 11 termos. E o melhor resultado do interpolar trigonométrico aconteceu para o caso de 7 termos.

- De todos os casos, a interpolação trigonométrica, sobre efemérides-fonte dadas no sistema inercial, foi o método que apresentou o melhor desempenho. Isto está ligado às características físicas dos dados (a natureza cíclica das órbitas GPS é mais bem representada por funções trigonométricas).

- Não foram percebidas diferenças significativas entre o mesmo procedimento de interpolação aplicado a diferentes satélites GPS (PRN 01 e $P R N 18)$.

\section{CONCLUSÕES}

A interpolação de efemérides GPS é necessária em vários tipos de aplicações: o posicionamento por ponto preciso (PPP precise point positioning) necessita de informações sobre a posição do satélite GPS e correção do relógio para as épocas de transmissão do sinal, que, quase sempre, não coincidem com a época nominal das efemérides produzidas pelo IGS e demais agências.

Para posicionamento preciso diferencial GPS em tempo real, em wide area, órbitas precisas, ou alternativamente, correções com respeito a uma órbita de referência (como as efemérides transmitidas) são necessárias (FENG e ZENG, 2005). Essas correções são geradas através de interpolações sobre efemérides, de forma similar ao efetuado neste trabalho, o que enfatiza a importância deste tipo de estudo.

Pelo fato das efemérides $I G S$ serem disponibilizadas em épocas nominais, igualmente espaçadas, o fenômeno de Runge está presente no início e final do 
conjunto de dados interpolados, sendo mais pronunciado nos 15 minutos finais do procedimento. Para evitar a perda de acurácia no processo de interpolação, esses extremos devem ser desprezados.

Os dois tipos de funções utilizadas se mostraram adequadas, mas a interpolação através de funções trigonométricas mostrou-se o método mais adequado a ser aplicado nas efemérides GPS disponibilizadas pelo IGS no formato SP3. Nesse caso o melhor desempenho do interpolador se deu com 7 termos, com as efemérides-fonte no sistema inercial.

Um método de interpolação não é considerado adequado se os erros de interpolação são maiores do que as incertezas nas efemérides. Escolhendo-se adequadamente o número de termos da função interpoladora, pode-se minimizar os erros de interpolação, tornando viável sua aplicação, como demonstram os resultados obtidos.

Dada a importância do tema "interpolação" dentro das Ciências Geodésicas, pretendeu-se com esse trabalho explicitar os algoritmos utilizados em SCHENEWERK (2003). Procurou-se ampliar os seus resultados com novos gráficos e análises.

Têm-se verificado que em alguns trabalhos em Ciências Geodésicas há alguma confusão entre os procedimentos de "interpolação" e "ajustamento", aparecendo algumas vezes o termo "interpolação" aplicado erroneamente no caso de ajustamento pelo método dos mínimos quadrados. Em análise numérica os procedimentos de interpolação e ajustamento são bastante distintos. Em virtude deste fato, procurou-se mostrar uma definição mais detalhada de interpolação, aplicada às Ciências Geodésicas.

\section{AGRADECIMENTOS:}

A primeira autora agradece ao CNPq pelo suporte financeiro.

\section{REFERÊNCIAS BIBLIOGRÁFICAS}

COSTA, M. A. F. Cálculo Numérico com Pascal. Lisboa: Escolar Editora, 1993.

FENG, Y. e ZHENG, Y. Efficient Interpolations to GPS Orbits for Precise Wide Area Applications. GPS Solutions, vol 9, pp 273-282, 2005.

GURTNER, W. Rinex: The Receiver Independent Exchange Format Version 2.1. Astronomical Institute, University of Berne, 08 de junho, 2001.

HILLA, S. The Extended Standard Product 3 Orbit Format (SP3c). National Geodetic Survey, Silver Spring, 12 de Fevereiro de 2007 (disponível em: $\mathrm{ftp} / / /$ igscb.jpl.nasa.gov/pub/data/format/sp3c.txt).

HOREMUZ, M. e ANDERSSON, J. V. Polynomial Interpolation of GPS Satellite Coordinates. GPS Solutions, vol. 10 , pp. 67-72, 2006.

IERS ANNUAL REPORT 2005. Verlag des Bundesamts für Kartographie und Geodäsie, Frankfurt am Main, 2007. 
IGS - THE INTERNATIONAL GPS SERVICE FOR LEADING-EDGE SPACE MISSIONS. ESA Bulletin 116, Darmstadt, Novembro de 2003. (disponível em: http://www.esa.int/esapub/bulletin/bullet116/chapter9_bul116.pdf)

KOUBA, J., MIREAUlT, Y., BEUTlER, G., SPRINGER, T., GENDT, G., $A$ Discussion of IGS Solutions And Their Impact On Geodetic And Geophysical Applications. GPS solutions 2(2), 3-15, 1998.

KREYSZIG, E. Advanced Engineering Mathematics. Nova York: Wiley Sons, 2006.

RALSTON, A. e RABINOWITZ, P. A First Course In Numerical Analysis. Nova York: Dover, 2001.

REMONDI, B., NGS SECOND GENERATION ASCII AND BINARY ORBIT FORMATS AND ASSOCIATED INTERPOLATION STUDIES. Apresentado na 20a. Assembléia Geral do IUGG, Viena, 11-24 de Agosto de 1991.

SCHENEWERK, M. A Brief Review Of Basic GPS Orbit Interpolation Strategies. GPS Solutions, vol. 6, pp. 265-267, 2003.

YOUNG, D. M. e GREGORY, R. T. A Survey Of Numerical Mathematics, vol. 1. Londres: Adisson-Wesley, 1972.

YOUSIF, H. e EL-RABBANY, A. Assessment Of Several Interpolation Methods for Precise GPS Orbit. The Journal Of Navigation, vol 60, pp 443-455, 2007.

(Recebido em julho de 2010. Aceito em junho de 2011). 